\title{
Reporting patient characteristics and stratification factors in randomized trials of systemic chemotherapy for advanced gastric cancer
}

\author{
Kohei Shitara $\cdot$ Junko Ikeda $\cdot$ Chihiro Kondo $\cdot$ \\ Daisuke Takahari $\cdot$ Takashi Ura $\cdot$ Kei Muro • \\ Keitaro Matsuo
}

Received: 14 May 2011/Accepted: 24 July 2011/Published online: 13 August 2011

(C) The International Gastric Cancer Association and The Japanese Gastric Cancer Association 2011

\begin{abstract}
Background There is no consensus on which patient characteristics are the most suitable to report or to be used as stratification factors in clinical trials for advanced gastric cancer (AGC), to our knowledge.

Methods We conducted a comprehensive review of published randomized trials for AGC to examine the patient characteristics that were reported.

Results Among the 67 analyzed trials, age, gender, performance status, proportion of patients with measurable disease, and previous gastrectomy were frequently reported $(>69 \%)$. Histology, number of disease sites, and adjuvant treatment were reported in less than $50 \%$ of trials. Although the reporting of second-line chemotherapy has increased in recent trials, it remains at less than $50 \%$. Notably, recent trials have tended to include patients with better performance status and less locally advanced disease, with Asian trials more frequently including patients with more diffuse histology and less locally advanced disease or liver metastasis than non-Asian trials. Stratification was conducted in approximately $60 \%$ of the trials, using quite variable stratifying factors.
\end{abstract}

Electronic supplementary material The online version of this article (doi:10.1007/s10120-011-0083-8) contains supplementary material, which is available to authorized users.

K. Shitara $(\bowtie) \cdot$ J. Ikeda $\cdot$ C. Kondo · D. Takahari · T. Ura · K. Muro

Department of Clinical Oncology, Aichi Cancer Center Hospital,

1-1 Kanokoden, Chikusa-ku, Nagoya, Aichi 464-8681, Japan

e-mail: Kouheis0824@yahoo.co.jp

K. Matsuo

Division of Epidemiology and Prevention, Aichi Cancer Center

Research Institute, Nagoya, Japan
Conclusion Inconsistency exists in the reporting of patient characteristics, the characteristics themselves, and the use of stratification factors in clinical trials for AGC. A consensus set of important patient characteristics and strata may be necessary to conduct and interpret quality randomized studies.

Keywords Chemotherapy - Gastric cancer · Prognostic factor $\cdot$ Randomized trial $\cdot$ Stratification

\section{Introduction}

Gastric cancer remains one of the most common malignancies and leading causes of cancer death worldwide [1]. Although the most effective treatment for localized disease is surgery, approximately half of all patients with advanced-stage disease experience recurrence following curative resection. The prognosis of patients with advanced or recurrent gastric cancer (AGC) remains poor, with commonly used combination chemotherapy regimens, consisting of a fluoropyrimidine plus a platinum agent with or without docetaxel or anthracyclines, leading to a median survival of only 1 year [2-8]. Therefore, the development of novel anticancer agents or strategies for the treatment for AGC is urgently required; however, for the evaluation of such agents and treatments, it is critical to conduct effective randomized trials.

Reflecting the relatively high incidence of gastric cancer worldwide, numerous clinical trials have been conducted in multiple countries or as part of global studies [7, 8]. These clinical trials have displayed surprising heterogeneity in overall survival (OS) even if patients with similar stages of unresectable AGC are targeted. Although several identified prognostic factors in patient characteristics and practice 
patterns, including surgery and chemotherapy, are thought to partially contribute to the observed heterogeneity [9], the exact reason for this heterogeneity is unknown.

A number of reports have evaluated prognostic factors in AGC patients who underwent chemotherapy [10-14]. For example, the recent Global Advanced/Adjuvant Stomach Tumor Research through International Collaboration (GASTRIC) project confirmed the impact of performance status (PS), disease status (metastatic vs. locally recurrence vs. locally advanced), number of metastatic organs, location of metastasis, and prior surgery on the survival of AGC based on individual patient data analysis of previous randomized studies [10]. In addition, Chau et al. [11] identified four independent prognostic factors for poor AGC survival: $\mathrm{PS} \geq 2$, liver metastasis, peritoneal metastasis, and increased serum alkaline phosphatase (ALP) levels, which were subsequently used to classify patients into three risk groups (Royal Marsden hospital prognostic index) that were validated in a large phase III trial [12]. The prognostic factors for AGC identified to date also serve as important stratification factors in randomized trials to exclude possible confounding variables. To our knowledge, however, there is no consensus as to the specific patient characteristics that are most suitable to report or to be used as stratification factors in clinical trials for AGC.

Here, we report the results of a comprehensive review of published randomized trials for AGC that we conducted to investigate the patient characteristics and stratification factors that have been evaluated and reported. We also examined differences in previous studies according to trial period and region.

\section{Materials and methods}

\section{Search for studies}

We conducted a literature search for randomized clinical trials of AGC through computer-based searches of the Medline database (January 1966 and December 2010) and searches of abstracts from conference proceedings of the American Society of Clinical Oncology (1995-2010), and the European Cancer Conference and European Society for Medical Oncology (1995-2010). Search key words included: "gastric cancer," "randomized", "advanced or metastatic", and "chemotherapy." The search was also guided by a thorough examination of reference lists from original and review articles.

\section{Procedures}

Two investigators (Kohei Shitara and Keitaro Matsuo) extracted data in accordance with the Quality of Reporting of Meta-analyses (QUORUM) guidelines [15]. Randomized trials of systemic chemotherapy for patients with histologically confirmed AGC (metastatic or unresectable locally advanced disease) of the stomach or gastroesophageal junction were included in the analyses. Trials that compared chemotherapy with best supportive care were also included, as were those which included patients with adenocarcinoma of the distal esophagus. Exclusion criteria included trials designed to assess combined modality treatments, including radiotherapy and surgery (neoadjuvant or adjuvant chemotherapies); and those in which patients were pretreated with systemic chemotherapy. Unpublished trials and trials published in non-English languages were also excluded from this analysis.

For each trial, the reporting of patient characteristics and stratification factors was extracted. As trial characteristics, the following information was extracted: first author's name, year of publication, trial design (randomized phase II or III, if reported), trial location, number of enrolled patients, and treatment regimens. As patient characteristics, the following information was extracted (if reported): age; gender; PS; histology (e.g., diffuse or intestinal type); disease status (e.g., advanced or recurrent disease); primary tumor location (e.g., stomach or gastroesophageal junction); extension of disease (e.g., locally advanced or metastatic); previous gastrectomy, adjuvant chemotherapy, and radiotherapy; sites of metastases (e.g., peritoneum, liver, and lymph node); number of metastatic organs; and proportion of patients with measurable disease. The proportion of patients who received second-line chemotherapy was also extracted. All data were checked for internal consistency.

\section{Statistical methods}

Differences in the reporting of patient characteristics according to trial period (before vs. after 2004) and trial region (Asian vs. non-Asian trials) were assessed by the $\chi^{2}$ test or Fisher's exact test, as appropriate. Because there was no definitive cut-off time for performing trend analysis, we divided the period at 2004 as this led to the number of trials (36 vs. 31 trials) and number of patients being almost equally distributed in the two periods. Median values for patient characteristics were calculated for each trial and the combined patient population. Differences in patient characteristics according to region or trial period were evaluated using the Mann-Whitney test. Use of stratification factors according to trial period or region was evaluated with the $\chi^{2}$ test or Fisher's exact test as appropriate. Statistical analyses were performed using STATA ver. 10 (StataCorp. LP; College Station, TX, USA). All tests were two-sided, and $P$ values of less than 0.05 were considered statistically significant. 


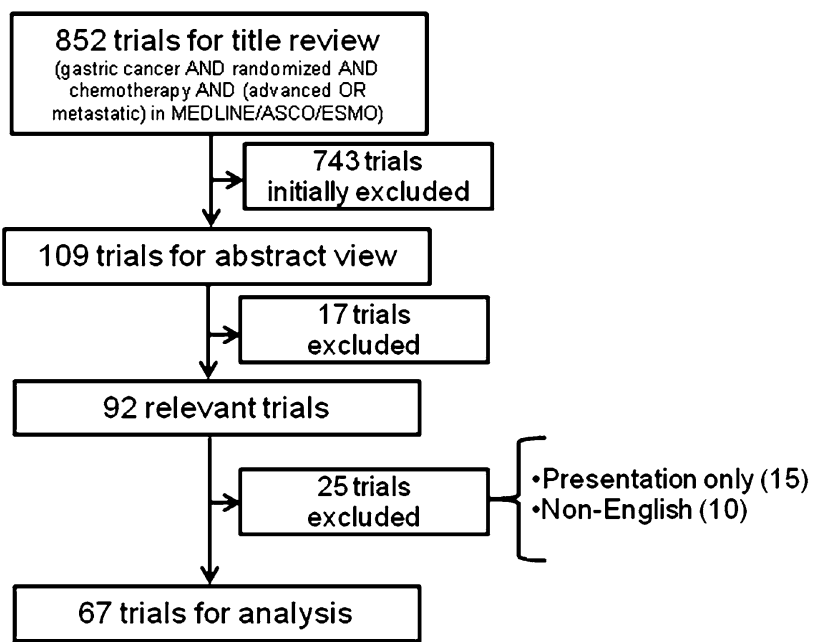

Fig. 1 Selection process for trials. An initial literature search for randomized clinical trials of advanced gastric cancer (AGC) identified a total of 852 potentially relevant reports, of which 743 were excluded on examination of titles. After review of the abstracts of the remaining studies, 67 randomized trials, with a total of 153 treatment arms and 12,656 patients were identified as eligible for analysis. ASCO American Society of Clinical Oncology, ESMO European Society for Medical Oncology

\section{Results}

\section{Study selection}

Our extensive literature search yielded a total of 852 potentially relevant reports, of which 743 were initially excluded on examination of titles (Fig. 1). After review of the abstracts of the remaining studies, 67 randomized trials, with a total of 153 treatment arms and 12,656 patients were identified as eligible for analysis (Supplement 1). Table 1 summarizes the characteristics of the 67 selected clinical trials, which consisted of 23 and 30 randomized phase II and III trials, respectively, and 14 trials that did not report the trial phase.

\section{Patient characteristics reported in trials}

Table 2 summarizes the patient characteristics reported in the 67 clinical trials included in the analysis. Two global studies that included Asian countries were excluded when comparing trials in Asia and non-Asian countries.

Age, gender, and PS

All 67 clinical trials provided information of patient age, with nearly all (94\%) providing a median value, and four trials providing categorized values. One trial targeted elderly patients (>70 years). Gender information was reported by all but one trial. Sixty-four trials (96\%) provided information regarding PS, with 46 reporting Eastern
Table 1 Characteristics of the 67 clinical trials analyzed in the present study

\begin{tabular}{lcc}
\hline Characteristic & $N$ & $\%$ \\
\hline Reported year & 36 & 54 \\
Before 2004 & 31 & 46 \\
$2004-2010$ & & \\
Trial setting & 23 & 34 \\
Phase II & 30 & 45 \\
Phase III & 14 & 21 \\
Not indicated & & \\
Number of patients & 28 & 42 \\
$<100$ & 28 & 42 \\
100-300 & 11 & 16 \\
$>300$ & & \\
Trial area & 14 & 21 \\
Asia & 12 & 18 \\
North America & 31 & 46 \\
Europe & 6 & 3 \\
Other & 2 & 3 \\
North America and Europe & 2 & \\
Global, including Asia & & \\
\hline
\end{tabular}

Cooperative Oncology Group (ECOG)/WHO PS classifications and the other 17 using the Karnofsky Performance Scale (KPS). Considerable PS variability was detected among the trial patients, as follows: PS 0-1, 4 trials; PS 0-2, 25 trials; and PS 0-3, 17 trials; and KPS 100-80, 1 trial; KPS 100-70, 5 trials; KPS 100-60, 7 trials; and KPS 100-50, 4 trials. Among the trials that used ECOG PS, 22 reported ECOG PS 0 versus 1 versus 2, whereas the other studies reported PS 0 and 1 without discrimination. No significant differences in reporting were detected in the trial period or region for PS, age, and gender.

\section{Disease characteristics}

The proportion of patients with measurable disease was reported in $69 \%$ of trials, with half including only patients with at least one measurable disease. Extension of disease and disease status were reported in 57 and $27 \%$ of trials, respectively. The location of metastases was reported in $64 \%$ of trials; the liver was the most commonly reported site, followed by the peritoneum. Histology and the number of metastatic organs were not reported in more than half of the trials. The Lauren classification (intestinal or diffuse type) was used in 12 trials, while classifications such as the American Joint Committee on Cancer grading system (well- or poorly differentiated adenocarcinoma, etc.) were used in 18 trials. The location of primary tumors was reported in 26 trials (39\%), with 17 trials including not only gastric cancer, but also esophagogastric junction or 
Table 2 Reported patient characteristics in the 67 clinical trials analyzed in the present study

\begin{tabular}{|c|c|c|c|c|c|c|c|}
\hline \multirow[t]{2}{*}{ Characteristic } & \multirow{2}{*}{$\begin{array}{l}\text { Reported } \\
\text { studies } \\
(\%)\end{array}$} & \multicolumn{3}{|l|}{ Reported year } & \multicolumn{3}{|c|}{ Area of trial ${ }^{\mathrm{a}}$} \\
\hline & & $\begin{array}{l}\text { Before } 2004 \\
(n=36)\end{array}$ & $\begin{array}{l}\text { After } 2004 \\
(n=31)\end{array}$ & $P$ value $^{\dagger}$ & $\begin{array}{l}\text { Non-Asian } \\
(n=51)\end{array}$ & $\begin{array}{l}\text { Asian } \\
(n=14)\end{array}$ & $P$ value $^{\dagger}$ \\
\hline Age & $67(100)$ & $36(100)$ & $31(100)$ & ns & $51(100)$ & $14(100)$ & ns \\
\hline Gender & $66(99)$ & $35(97)$ & $31(100)$ & ns & $50(98)$ & $14(100)$ & ns \\
\hline PS & $64(96)$ & $34(94)$ & $30(97)$ & $\mathrm{ns}$ & $48(94)$ & $14(100)$ & ns \\
\hline Measurable disease & $46(69)$ & $21(58)$ & $25(81)$ & $\underline{0.05}$ & $35(69)$ & $9(64)$ & ns \\
\hline Metastatic site & $43(64)$ & $22(61)$ & $21(68)$ & ns & $33(65)$ & $9(64)$ & ns \\
\hline Disease extension (local or metastatic) & $38(57)$ & $19(53)$ & $19(61)$ & ns & $33(65)$ & $5(36)$ & ns \\
\hline Histology & $30(45)$ & $12(33)$ & $18(58)$ & $\underline{0.04}$ & $20(39)$ & $9(64)$ & ns \\
\hline Location of primary tumor & $26(39)$ & $8(22)$ & $18(58)$ & $\leq \underline{0.01}$ & $24(47)$ & $1(7)$ & $\leq \underline{0.01}$ \\
\hline Number of metastatic organs & $25(37)$ & $5(14)$ & $20(65)$ & $\leq \underline{0.01}$ & $18(35)$ & $5(36)$ & ns \\
\hline Disease status (advanced or recurrent) & $18(27)$ & $5(14)$ & $13(42)$ & $\mathrm{ns}$ & $13(25)$ & $5(36)$ & ns \\
\hline Previous gastrectomy & $46(69)$ & $21(58)$ & $25(81)$ & $\underline{0.05}$ & $32(63)$ & $12(86)$ & ns \\
\hline Previous adjuvant chemotherapy & $16(24)$ & $0(0)$ & $16(52)$ & $\leq \underline{0.01}$ & $6(12)$ & $9(64)$ & $\leq \underline{0.01}$ \\
\hline Previous radiotherapy & $11(16)$ & $3(8)$ & $8(26)$ & ns & $9(17)$ & $1(7)$ & ns \\
\hline Second-line chemotherapy & $18(27)$ & $3(8)$ & $15(48)$ & $\leq 0.01$ & $10(20)$ & $6(43)$ & ns \\
\hline
\end{tabular}

ns not significant, $P S$ performance status

${ }^{a}$ Excluded two global studies

${ }^{\dagger}$ Statistical analyses were performed using the $\chi^{2}$ test or Fisher's exact test, with the level of significance set at $P<0.05$ (underlined)

esophageal cancer. The frequency of reporting these characteristics appeared to be increasing in more recent trials, although most examined characteristics were reported in less than $60 \%$ of the trials (Table 2). Only primary tumor location was more frequently reported in non-Asian than Asian trials, and no other significant differences in reporting of disease characteristics were observed based on trial area.

The other reported patient characteristics were as follows: weight loss $(n=12 ; 18 \%)$; any symptoms (anorexia, dysphasia, etc., $n=7 ; 10 \%)$; body surface area $(n=3 ; 4 \%)$; ethnic groups $(n=2 ; 3 \%)$; hemoglobin level $(n=4 ; 6 \%)$; serum ALP level $(n=3 ; 4 \%)$; comorbidities $(n=3 ; 4 \%)$, and Royal Marsden hospital prognostic index $(n=1 ; 1 \%)$.

Previous treatment and second-line chemotherapy

An indication of the proportion of patients with previous gastrectomy was reported in $69 \%$ of trials, with the curability of gastrectomy (curative or palliative with residual disease) specified in approximately $50 \%$ of trials. Previous adjuvant chemotherapy and radiotherapy were infrequently reported (24 and $16 \%$ of trials, respectively). Second-line chemotherapy was also reported with low frequency (27\% of trials), and was typically indicated in the text, rather than being included in patient characteristic tables. The reporting of previous treatment and second-line chemotherapies was found to be increasing in recent trials, although more than half did not include information related to second-line chemotherapy. In addition, Asian trials more commonly reported the use of adjuvant chemotherapy than non-Asian trials.

Patient characteristics of the combined trial population

The characteristics of the 12,656 AGC patients were calculated based on the reported values in each of the 67 clinical trials (Table 3). Recent trials included more patients with better PS (ECOG PS $0-1 ; 94$ vs. 64\%; $P<0.01)$ and less locally advanced disease (4 vs. $27 \%$ ) than older trials. Asian trials included more patients with diffuse histology than non-Asian trials (53 vs. 34\%; $P<0.01$ ), while patients with liver metastasis (43 vs. $31 \% ; P=0.01)$ or locally advanced disease (15 vs. $3 \%$; $P=0.04)$ were more common in non-Asian trials. Secondline chemotherapy was more commonly used in Asian and recent trials.

\section{Stratification factors}

Among the 67 trials, $40(60 \%)$ used stratification factors (Table 4). The median number of factors was 3 , with an observed range of $1-5$. The most common stratification factor was PS, followed by institution and previous gastrectomy. More recent trials used one or more stratification factors than older trials ( 47 vs. $75 \%, P=0.03$, Table 4 ). 
Table 3 Patient characteristics $(n=12,656)$ in AGC trials included in this analysis

\begin{tabular}{|c|c|c|c|c|c|c|c|c|c|}
\hline \multirow[t]{2}{*}{ Patient characteristic } & \multirow{2}{*}{$\begin{array}{l}\text { Entire patient } \\
\text { population } \\
\text { (median) }\end{array}$} & \multirow{2}{*}{$\begin{array}{l}\text { Median } \\
\text { per trial }\end{array}$} & \multirow[t]{2}{*}{ Range } & \multicolumn{3}{|c|}{ Reported year } & \multicolumn{3}{|c|}{ Area of trial ${ }^{\mathrm{a}}$} \\
\hline & & & & $\begin{array}{l}\text { Before } 2004 \\
\text { (median) }\end{array}$ & $\begin{array}{l}\text { After } 2004 \\
\text { (median) }\end{array}$ & $P$ value $^{\dagger}$ & $\begin{array}{l}\text { Non-Asian } \\
\text { (median) }\end{array}$ & $\begin{array}{l}\text { Asian } \\
\text { (median) }\end{array}$ & $P$ value $^{\dagger}$ \\
\hline Median age (years) & - & 59 & $52-72$ & 58 & 59 & $\mathrm{~ns}$ & 59 & 58 & Ns \\
\hline Male gender $(\%)$ & 73 & 72 & $58-83$ & 70 & 74 & $\mathrm{~ns}$ & 72 & 69 & ns \\
\hline PS0-1 (\%) & 84 & 83 & $18-100$ & 69 & 94 & $\leq \underline{0.01}$ & 78 & 89 & ns \\
\hline PS 2 or more $(\%)$ & 16 & 17 & $0-82$ & 31 & 6 & $\leq 0.01$ & 22 & 11 & ns \\
\hline Diffuse histology (\%) & 42 & 38 & $1-66$ & 44 & 34 & ns & 34 & 53 & $\leq \underline{0.01}$ \\
\hline One metastatic organ $(\%)$ & 33 & 30 & $9-51$ & 26 & 32 & $\mathrm{~ns}$ & 27 & 35 & ns \\
\hline Locally advanced disease (\%) & 15 & 14 & $0-43$ & 27 & 4 & $\leq 0.01$ & 15 & 3 & 0.04 \\
\hline Liver metastasis (\%) & 44 & 42 & $18-79$ & 42 & 42 & $\mathrm{~ns}$ & 43 & 31 & $\underline{0.02}$ \\
\hline Peritoneal metastasis (\%) & 23 & 24 & $3-62$ & 23 & 29 & $\mathrm{~ns}$ & 20 & 29 & ns \\
\hline With measurable disease $(\%)$ & 88 & 99 & $33-100$ & 96 & 100 & $\mathrm{~ns}$ & 100 & 96 & ns \\
\hline Previous gastrectomy (\%) & 33 & 39 & $8-83$ & 38 & 40 & ns & 41 & 33 & ns \\
\hline $\begin{array}{l}\text { Previous adjuvant } \\
\text { chemotherapy }(\%)\end{array}$ & 5 & 5 & $1-31$ & - & 5 & - & 4 & 9 & 0.02 \\
\hline Previous radiotherapy $(\%)$ & 1 & 1 & $0-3$ & 2 & 1 & $\mathrm{~ns}$ & 1 & 1 & ns \\
\hline Second-line chemotherapy (\%) & 40 & 41 & $14-83$ & 18 & 40 & $\leq \underline{0.01}$ & 36 & 57 & 0.01 \\
\hline
\end{tabular}

$n s$ not significant, $P S$ performance status

${ }^{a}$ Excluded two global studies

${ }^{\dagger}$ Statistical analyses were performed using the Mann-Whitney test, with the level of significance set at $P<0.05$ (underlined)

Table 4 Stratification factors in the 67 clinical trials analyzed in the present study

\begin{tabular}{|c|c|c|c|c|c|c|c|}
\hline \multirow[t]{2}{*}{ Stratification factor } & \multirow[t]{2}{*}{$N$ of studies $(\%)$} & \multicolumn{3}{|l|}{ Reported year } & \multicolumn{3}{|l|}{ Area of trial ${ }^{\mathrm{a}}$} \\
\hline & & Before $2004(\%)$ & After $2004(\%)$ & $P$ value $^{\dagger}$ & Non-Asian (\%) & $\begin{array}{l}\text { Asian } \\
(\%)\end{array}$ & $P$ value $^{\dagger}$ \\
\hline No factor & $27(47)$ & $19(53)$ & $8(26)$ & $\underline{0.03}$ & $22(43)$ & $5(36)$ & ns \\
\hline 1 or 2 factors & $12(21)$ & $5(14)$ & $7(23)$ & & $7(14)$ & $4(29)$ & \\
\hline 3 or more factors & $28(49)$ & $12(33)$ & $16(52)$ & & $22(43)$ & $5(36)$ & \\
\hline PS & $24(42)$ & $9(25)$ & $15(48)$ & ns & $16(31)$ & $7(50)$ & ns \\
\hline Previous gastrectomy & $18(32)$ & $9(25)$ & $9(29)$ & ns & $14(27)$ & $4(29)$ & ns \\
\hline Institution & $18(32)$ & $5(14)$ & $7(23)$ & 0.35 & $16(31)$ & $2(14)$ & ns \\
\hline Measurable disease & $12(21)$ & $6(17)$ & $6(19)$ & ns & $10(20)$ & $1(7)$ & ns \\
\hline Metastatic sites & $8(14)$ & $2(6)$ & $6(19)$ & 0.08 & $8(16)$ & $0(0)$ & ns \\
\hline Disease extension & $8(14)$ & $4(11)$ & $4(13)$ & ns & 7 (14) & $1(7)$ & ns \\
\hline Age & $6(11)$ & $5(14)$ & $1(3)$ & ns & $5(10)$ & $1(7)$ & ns \\
\hline Gender & $5(9)$ & $5(14)$ & $0(0)$ & $\underline{0.03}$ & $5(10)$ & $0(0)$ & ns \\
\hline Adjuvant chemotherapy & $5(9)$ & $1(3)$ & $4(13)$ & ns & $3(6)$ & $2(14)$ & ns \\
\hline Disease status & $3(5)$ & $0(0)$ & $3(10)$ & ns & $0(0)$ & $2(14)$ & $\leq \underline{0.01}$ \\
\hline Location of primary tumor & $3(5)$ & $1(3)$ & $2(6)$ & ns & $2(4)$ & $0(0)$ & ns \\
\hline
\end{tabular}

$n s$ not significant, $P S$ performance status

${ }^{a}$ Excluded two global studies

$\dagger$ Statistical analyses were performed using the $\chi^{2}$ test or Fisher's exact test, with the level of significance set at $P<0.05$ (underlined)

Gender was more commonly used in older trials (14 vs. $0 \%$ ). No significant difference of stratification factors was observed between Asian and non-Asian trials, other than the frequency of use of disease status (0 vs. 14\%).

\section{Discussion}

To our knowledge, this represents the first study to review the reporting of patient characteristics in published 
randomized trials for AGC. Our results showed considerable inconsistency in the reporting of patient characteristics and the use of stratification factors in clinical trials for AGC. A similar finding was reported by Sorbye et al. [16], who analyzed metastatic colorectal cancer (MCRC) clinical trials and advocated that an urgent need exists for an international consensus on the reporting of patient characteristics and stratification in MCRC trials. Our data also revealed several differences in patient characteristics between trials conducted before and after 2004, and between Asian and non-Asian trials. It is possible that these differences may have contributed to the observed heterogeneity in the survival outcomes of each trial.

Several prognostic factors have been identified for patients with AGC who have undergone chemotherapy [10-14]. As described in the "Introduction", the GASTRIC project confirmed the impact of ECOG PS, disease status, number of metastatic organs, location of metastasis, and prior surgery on the survival of AGC patients, as determined by individual patient data analysis of previous randomized studies [10]. Notably, this project, which may have included the largest AGC patient set to date, identified that PS1 and PS2 were significantly associated with poor survival, with hazard ratios (HRs) of death of 1.36 and 2.17, respectively [10]. In the GASTRIC analysis, although most trials included PS among the reported patient characteristics, a number of studies classified PS0 and PS1 separately, and several studies used KPS rather than the ECOG scale. In addition, local recurrence and metastatic disease were reported to be associated with worse outcomes than locally advanced disease [10]. In our present analysis, approximately $50 \%$ of trials reported disease extension (locally advanced or metastatic disease), and only $30 \%$ of trials indicated disease status (advanced or recurrent disease).

Although the GASTRIC analysis did not evaluate the importance of specific metastatic organs on outcomes, another large prognostic analysis, by Chau et al. [11, 12], reported the impact of liver and peritoneal metastasis on AGC patient survival. Affected metastatic organs were reported in $64 \%$ of the trials in our analysis, but the number of metastatic organs, which has significant impact on survival according to the GASTRIC analysis, was only reported with a frequency of $39 \%$. Although histology was not identified as prognostic in the GASTRIC analysis, several recent trials suggest that an interaction exists between histology and drug response $[6,7,17,18]$. For example, a subset analysis of the First-line Advanced Gastric Cancer Study (FLAGS) trial has indicated that the oral fluoropyrimidine S-1 appears to be superior to fluorouracil in the treatment of diffuse-type gastric cancer [6]. This finding is consistent with the results of a subset analysis of the Japan Clinical Oncology Group (JCOG) 9912 study that also indicated S-1 is better than fluorouracil in patients with diffuse-type AGC or gastric cancer associated with high dihydropyrimidine dehydrogenase (DPD) activity, which is more commonly associated with diffuse-type than intestinal-type tumors [17]. This result was not unexpected, because S-1 is a potent competitive inhibitor of DPD. In contrast to DPD, human epidermal growth factor receptor 2 (HER2)-positive AGC, for which the anti-HER2 agent trastuzumab is effective [7], is reported to be higher among intestinal-type tumors [18]. The prognostic factors and tumor characteristics identified in these studies should be reported in all clinical trials of AGC, as they are necessary to adequately interpret trial data and treatment outcomes.

Our analysis also revealed that the types of second-line chemotherapy and proportions of patients who received such treatment were not routinely reported in AGC trials. As several recent reports have suggested that second-line chemotherapy has a significant impact on OS [19-21], we propose that second-line therapies should be diligently reported in future clinical trials of first-line AGC treatment, because second-line chemotherapy might influence the OS as the primary endpoint, as suggested by our previous analysis [22].

Additionally, the numerous prognostic factors identified for AGC may be important for the stratification of patients with respect to risk and treatment arms in randomized trials. To adequately analyze treatment effects on clinical outcomes, efforts should be undertaken to maximally decrease imbalance of prognostic factors between treatment arms in a clinical trial [23]. Although there is no definite consensus on the optimal method for stratification, stratification is recommended for superiority trials with fewer than 400 patients [24] and for non-inferiority trials with any number of patients [25]. In our analysis, stratification was conducted in only $60 \%$ of the examined trials, and was performed with quite variable stratifying factors. Based only on the present analysis, it is difficult to suggest a standardization approach for stratification factors in AGC trials, and further analysis and discussion are necessary.

In recent years, a trend of increased median OS in AGC patients has been observed concurrent with the development of new chemotherapeutic agents $[2,4,7,26]$. It is also possible that second-line chemotherapy may have contributed to the improvement in OS [19-21]; however, our crude comparison of trials conducted prior to and after 2004 also showed significant differences in PS and disease extension. These differences may have also contributed to the improved survival reported in more recent trials, as well as survival differences between Asian and non-Asian trials. The exact impact of chemotherapy and patient characteristics on survival would be best addressed in well-designed randomized studies and meta-analyses of individual patient data.

In conclusion, our analyses of published clinical trials for AGC revealed inconsistencies in the reporting of 
patient characteristics and use of stratification factors. An international consensus on the reported characteristics and stratification in AGC trials is necessary to improve the analysis of future clinical trials.

Conflict of interest None.

\section{References}

1. Kamangar F, Dores GM, Anderson WF. Patterns of cancer incidence, mortality, and prevalence across five continents: defining priorities to reduce cancer disparities in different geographic regions of the world. J Clin Oncol. 2006;24:2137-50.

2. Van Cutsem E, Moiseyenko VM, Tjulandin S, Majlis A, Constenla M, Boni C, et al. Phase III study of docetaxel and cisplatin plus fluorouracil compared with cisplatin and fluorouracil as firstline therapy for advanced gastric cancer: a report of the V325 Study Group. J Clin Oncol. 2006;24:4991-7.

3. Cunningham D, Starling N, Rao S, Iveson T, Nicolson M, Coxon F, et al. Capecitabine and oxaliplatin for advanced esophagogastric cancer. N Engl J Med. 2008;358:36-46.

4. Koizumi W, Narahara H, Hara T, Takagane A, Akiya T, Takagi M, et al. S-1 plus cisplatin versus S-1 alone for first-line treatment of advanced gastric cancer (SPIRITS trial): a phase III trial. Lancet Oncol. 2008;9:215-21.

5. Kang YK, Kang WK, Shin DB, Chen J, Xiong J, Wang J, et al. Capecitabine/cisplatin versus 5-fluorouracil/cisplatin as first-line therapy in patients with advanced gastric cancer: a randomised phase III noninferiority trial. Ann Oncol. 2009;20:666-73.

6. Ajani JA, Rodriguez W, Bodoky G, Moiseyenko V, Lichinitser $\mathrm{M}$, Gorbunova V, et al. Multicenter phase III comparison of cisplatin/S-1 with cisplatin/infusional fluorouracil in advanced gastric or gastroesophageal adenocarcinoma study: the FLAGS trial. J Clin Oncol. 2010;28:1547-53.

7. Bang YJ, Van Cutsem E, Feyereislova A, Chung HC, Shen L, Sawaki A, et al. Trastuzumab in combination with chemotherapy versus chemotherapy alone for treatment of HER2-positive advanced gastric or gastro-oesophageal junction cancer (ToGA): a phase 3, open-label, randomised controlled trial. Lancet. 2010;376:687-97.

8. Kang Y, Ohtsu A, Van Cutsem E, Roman L, Nunes J, Li C, et al. AVAGAST: a randomized, double-blind, placebo-controlled, phase III study of first-line capecitabine and cisplatin plus bevacizumab or placebo in patients with advanced gastric cancer (AGC). J Clin Oncol. 2010;28:18s (suppl; abstr LBA4007).

9. Ohtsu A. Diverse Eastern and Western approaches to the management of gastric cancer. Gastrointest Cancer Res. 2007;1:S10-5.

10. Pozzo C, Ohashi Y, on behalf of the GASTRIC project. Metaanalyses of randomized trials assessing the influence of chemotherapy and prognostic factor in advanced/recurrent gastric cancer. J Clin Oncol. 2009;27:15s (suppl; abstr 4550).

11. Chau I, Norman AR, Cunningham D, Waters JS, Oates J, Ross PJ. Multivariate prognostic factor analysis in locally advanced and metastatic esophago-gastric cancer. Pooled analysis from three multicenter, randomized, controlled trials using individual patient data. J Clin Oncol. 2004;22:2395-403.

12. Chau I, Ashley S, Cunningham D. Validation of the Royal Marsden hospital prognostic index in advanced esophagogastric cancer using individual patient data from the REAL 2 study. J Clin Oncol. 2009;27:e3-4.

13. Yoshida M, Ohtsu A, Boku N, Miyata Y, Shirao K, Shimada Y, et al. Long-term survival and prognostic factors in patients with metastatic gastric cancers treated with chemotherapy in the Japan Clinical Oncology Group (JCOG) study. Jpn J Clin Oncol. 2004;34:654-9.

14. Kim JG, Ryoo BY, Park YH, Kim BS, Kim TY, Im YH, et al. Prognostic factors for survival of patients with advanced gastric cancer treated with cisplatin-based chemotherapy. Cancer Chemother Pharmacol. 2008;61:301-7.

15. Hopewell S, Clarke M, Moher D, Wager E, Middleton P, Altman DG, for the CONSORT group, et al. CONSORT for reporting randomised trials in journal and conference abstracts. Lancet. 2008;371: 281-83.

16. Sorbye H, Köhne CH, Sargent DJ, Glimelius B. Patient characteristics and stratification in medical treatment studies for metastatic colorectal cancer: a proposal for standardization of patient characteristic reporting and stratification. Ann Oncol. 2007;18: 1666-72.

17. Yamada Y, Yamamoto S, Ohtsu A, Suzuki Y, Nasu J, Yamaguchi $\mathrm{K}$, et al. Impact of dihydropyrimidine dehydrogenase status of biopsy specimens on efficacy of irinotecan plus cisplatin, S-1, or 5 -FU as first-line treatment of advanced gastric cancer patients in JCOG9912. J Clin Oncol. 2009;27:15s (suppl; abstr 4535).

18. Bang Y, Chung H, Sawaki A, Xu J, Shen L, Lipatov O, et al. Pathological features of advanced gastric cancer (GC): relationship to human epidermal growth factor receptor 2 (HER2) positivity in the global screening programme of the ToGA trial. J Clin Oncol. 2009;27:15s (suppl; abstr 4556).

19. Thuss-Patience PC, Kretzschmar A, Deist T, Hinke A, Bichev D, Lebedinzew B, et al. Irinotecan versus best supportive care (BSC) as second-line therapy in gastric cancer: a randomized phase III study of the Arbeitsgemeinschaft Internistische Onkologie (AIO). J Clin Oncol. 2009;27:15s (suppl; abstr 4540).

20. Takiuchi H, Fukuda H, Boku N, Shimada Y, Nasu J, Hamamoto $\mathrm{Y}$, et al. Randomized phase II study of best-available 5-fluorouracil (5-FU) versus weekly paclitaxel in gastric cancer (GC) with peritoneal metastasis (PM) refractory to 5-FU-containing regimens (JCOG0407). J Clin Oncol. 2010;28:15s (suppl; abstr 4052).

21. Takashima A, Boku N, Kato K, Mizusawa J, Nakamura K, Fukuda H, et al. Survival prolongation after treatment failure in patients with advanced gastric cancer (AGC): results from combined analysis of JCOG9205 and JCOG9912. J Clin Oncol. 2010;28:15s (suppl; abstr 4061).

22. Shitara K, Ikeda J, Yokota T, Takahari D, Ura T, Muro K, et al. Progression-free survival and time to progression as surrogate markers of overall survival in patients with advanced gastric cancer: analysis of 36 randomized trials. Invest New Drugs. 2011;Epub ahead of print.

23. Bajorin D. The phase III candidate: can we improve in the science of selection? J Clin Oncol. 2004;22:211-3.

24. Kernan WN, Viscoli CM, Makuch RW, Brass LM, Horwitz RI, et al. Stratified randomization for clinical trials. J Clin Epidemiol. 1999;52:19-26.

25. Nam JM. Sample size determination in stratified trials to establish the equivalence of two treatments. Stat Med. 1995;14:2037-49.

26. Wagner AD, Unverzagt S, Grothe W, Kleber G, Grothey A, Haerting J, et al. Chemotherapy for advanced gastric cancer. Cochrane Database Syst Rev. 2010;3:CD004064. 